\title{
TRPIV investigation of space-time correlation in turbulent flows over flat and wavy walls
}

\author{
Wei Wang · Xin-Lei Guan · Nan Jiang
}

Received: 21 October 2013 / Revised: 19 December 2013 / Accepted: 3 January 2014

(C)The Chinese Society of Theoretical and Applied Mechanics and Springer-Verlag Berlin Heidelberg 2014

\begin{abstract}
The present experimental work is devoted to investigate a new space-time correlation model for the turbulent boundary layer over a flat and a wavy walls. A turbulent boundary layer flow at $R e_{\theta}=2460$ is measured by tomographic time-resolved particle image velocimetry (Tomo-TRPIV). The space-time correlations of instantaneous streamwise fluctuation velocity are calculated at 3 different wall-normal locations in logarithmic layer. It is found that the scales of coherent structure increase with moving far away from the wall. The growth of scales is a manifestation of the growth of prevalent coherent structures in the turbulent boundary layer like hairpin vortex or hairpin packets when they lift up. The resulting contours of the space-time correlation exhibit elliptic-like shapes rather than straight lines. It is suggested that, instead of Taylor hypothesis, the elliptic model of the space-time correlation is valid for the wallbounded turbulent flow over either a flat wall or a wavy wall. The elliptic iso-correlation curves have a uniform preferred
\end{abstract}

The project was supported by the National Natural Science Foundation of China (11332006 and 11272233), the National Key Basic Research Program (2012CB720101), Tianjin University Research and Innovation Foundation and the opening subjects of The State Key Laboratory of Nonlinear Mechanics (LNM), Institute of Mechanics, Chinese Academy of Sciences.

W. Wang $\cdot$ X.-L. Guan $\cdot$ N. Jiang $(\bowtie)$

Department of Mechanics,

Tianjin University, 300072 Tianjin, China

e-mail: nanj@tju.edu.cn

\section{N. Jiang}

Tianjin Key Laboratory of Modern Engineering Mechanics, 300072 Tianjin, China

The State Key Laboratory of Nonlinear Mechanics, Institute of Mechanics, Chinese Academy of Sciences, 100190 Beijing, China orientation whose slope is determined by the convection velocity. The convection velocity derived from the space-time correlation represents the velocity at which the large-scale eddies carry small-scale eddies. The sweep velocity represents the distortions of the small-scale eddies and is intimately associated with the fluctuation velocity in the logarithmic layer of turbulent boundary layers. The nondimensionalized correlation curves confirm that the elliptic model is more proper for approximating the space-time correlation than Taylor hypothesis, because the latter can not embody the small-scale motions which have non-negligible distortions. A second flow over a wavy wall is also recorded using TRPIV. Due to the combined effect of shear layers and the adverse pressure gradient, the space-time correlation does not show an elliptic-like shape at some specific heights over the wavy wall, but in the outer region of the wavy wallbounded flow, the elliptic model remains valid.

Keywords Tomographic particle image velocimetry · Turbulent boundary layer · Wavy wall $\cdot$ Space-time correlation . Taylor hypothesis $\cdot$ Elliptic model

\section{Introduction}

Turbulence contains manifold scale of eddies, and a turbulence kinetic energy cascade model was proposed by Kolmogorov [1], which is usually characterized by frequency or wavenumber spectrum. The space-time correlations are the mostly usual statistics of turbulence. They have received constant attentions due to their value both in turbulence fundamental researches [2] and engineering applications. The space-time correlations of the velocity fluctuations are very useful inputs for prediction in the control and drag reduction of turbulence [3].

To obtain complete space-time information simultaneously, experimenters must face a severe challenge for sufficient resolution both in spatial and temporal scale. Hotwire anemometry has the advantage of high time-resolution 
and can easily infer the frequency spectrum. But hot-wire velocimetry is pointwise and can not obtain simultaneous velocity distribution in space. Taylor [4] proposed the famous frozen hypothesis by which we can derive the space correlations from the time correlations. Taylor frozen hypothesis has been extensively applied to obtain experimental estimates of dissipation rates and vorticity. Alternative application is in the study of coherent structures to obtain their properties such as convection velocity and so on. But accompanying its wide applications, doubts about its validity accumulates. Taylor frozen hypothesis suggests that the spatial patterns in turbulent flows are passing a fixed point without any essential change. "Without change" means that the motions drifting in the flow behave as being frozen which is the weakness of the hypothesis. Researchers have put forward several premises on which Taylor hypothesis can be successfully employed. For example, Lin [5] pointed out that the turbulence intensity must be low enough, viscous forces were negligible and the mean shear was small, all of which are requisite to make the frozen situation valid. Another problem arising from Taylor hypothesis is that the mean flow velocity is considered as the convection velocity for all scales of eddies which is too crude to specify multi-scale eddy motions in a turbulent flow.

The classical Taylor hypothesis simplifies the spacetime correlation to the space correlation via

$C(r, \tau)=C(r-U \tau, 0)$,

where $C$ is the correlation function normalized by the root mean square (r.m.s) of the velocity fluctuation, $r$ is the space separation, $\tau$ is the time delay and $U$ is the local longitudinal mean velocity. Taylor hypothesis is a first-order approximation for the space-time correlation. Its iso-correlation lines are straight lines in the space-time domain with its slope equal to the convection velocity $U$. When the space separation becomes large, the correlations remain unchanged. Such behavior violates the principle that correlations should decay with increasing separation. He et al. [6] and Zhao et al. [7] advance a second-order approximation model for the spacetime correlation. They relate the space-time correlation to the space correlation via

$C(r, \tau)=C\left(\sqrt{\left(r-U_{\mathrm{c}} \tau\right)^{2}+(V \tau)^{2}}, 0\right)$,

where $U_{\mathrm{c}}$ is the convection velocity and $V$ is the sweep velocity. The convection velocity $U_{\mathrm{c}}$ represents the velocity at which the large-scale eddies carry the small scale eddies. The sweep velocity $V$ represents the distortions of the small scale eddies. When $V$ vanishes, Eq. (2) is reduced to Eq. (1), which means that the Taylor hypothesis is indeed a simplified case of the new model. When $U_{\mathrm{c}}$ vanishes, Eq. (2) is actually the random sweeping-velocity hypothesis $[8,9]$. Equation (2) indicates that the iso-correlation lines follow the equation below,

$\left(r-U_{\mathrm{c}} \tau\right)^{2}+(V \tau)^{2}=r_{\mathrm{E}}^{2}$, which implies an elliptic shape for every iso-correlation contour. So we can call the new approximation of the space-time correlation "elliptic model". There are two recognizable characters in these elliptic iso-correlation curves. The first, all the iso-correlation curves share a uniform preferred orientation. And the slope of the preferred orientation is mainly determined by the convection velocity $U_{\mathrm{c}}$. The second, all the iso-correlation curves have a constant aspect ratio which can be derived from the Kolmogorov similar hypothesis.

He et al. [6] and Zhao et al. [7] verified the elliptic model using data obtained by simulating a homogeneous shear channel flow. The space-time correlations derived from the channel flow displayed elliptic-like contours. And the distributions of characteristic parameters, such as convection velocity, sweep velocity and so on, were consistent with their theoretical results. Recently Zhou et al. [10] experimentally verified the elliptic model in a turbulent RayleighBénard convection. While Taylor hypothesis was found invalid in the thermal convection, the correlations supported the elliptic model too.

The aim of the present work is to verify the elliptic model in turbulent boundary layer where the shear is nonhomogeneous. The experimental data were acquired by tomographic time-resolved particle image velocimetry (TomoTRPIV) in a turbulent boundary layer over either a flat wall and a wavy wall. Experimental details are described in Sect. 2. In Sect. 3.1 we briefly illustrate the mean flow fields of the turbulent boundary layer, and then calculate the spacetime correlation in Sect. 3.2. We focus on the characteristic parameters in the elliptic model in Sect.3.3. We compare the nondimensionalized correlation curves between the Taylor model and the elliptic model. In Sect. 4 we verify the elliptic model in flow over a wavy wall, which has a distinctive nature of turbulent production from the canonical turbulent boundary layer. Finally, conclusions are drawn in Sect. 5.

\section{Experimental set-up}

The first experiment was conducted in the water tunnel of the Laboratory for Aero and Hydrodynamics at TU Delft. The turbulent boundary layer data was obtained using TomoTRPIV [11, 12]. The data was first presented by Schröder et al. [13] to study the coherent structures in turbulent boundary layers. The basic properties of TBL are listed in Table 1. A more detailed set of experimental parameters was delineated in Schröder et al. [13].

The second experiment was conducted in a circulating water channel of Fluid Dynamic Laboratory at Tianjin University with a test section of $1300 \mathrm{~mm}$ (length) $\times 140 \mathrm{~mm}$ (width) $\times 150 \mathrm{~mm}$ (depth) using a 2D planar-TRPIV system, as shown in Fig. 1. The coordinates in both experiments, i.e., $x, y$, and $z$, correspond respectively to the streamwise, wall-normal and spanwise directions and so do the components of velocities, $u, v$, and $w$. The field of view was illuminated by a $532 \mathrm{~nm}$ green laser sheet which approximately lied in the spanwise center line of the channel. A high-speed 
camera $(1280 \times 1024$ pixels $)$ recorded particle images from the side view (streamwise-wall normal plane) with the beginning of images located at 32th peak of the wavy wall, about $70 \mathrm{~cm}$. There were 6400 snapshots of particle images that were recorded at $600 \mathrm{~Hz}$ sampling frequency. Due to the reflection of the wall surface, background subtraction was first employed to eliminate the background disturbance. Then an interrogation window of $32 \times 32$ pixels was adopted in an adaptive correlation process for determining the 2 components of velocities in the plane. With an overlap rate of $75 \%$, the measured fields consisted of $157 \times 125$ velocity vectors whose separations were $0.7226 \mathrm{~mm}(13.4 \mathrm{WU})$ in both directions. After the correlation process, a combination of range validation and average filter was applied for further removing the noise and increasing the number of effective velocity vectors. The profile of the wavy wall was fitted by $7.4+1.5 \cos (0.296 x-1.55)$, having a $21.6 \mathrm{~mm}$ wavelength with $3 \mathrm{~mm}$ wave-amplitude. The free-stream velocity $U_{\mathrm{e}}$ is $0.23 \mathrm{~m} / \mathrm{s}$, corresponding to a nominal boundary layer thickness Reynolds number $\operatorname{Re}_{\delta}=7480$.

Table 1 Basic properties of TBL over a flat wall

\begin{tabular}{ll}
\hline Free-stream velocity $U_{\mathrm{e}}$ & $0.53 \mathrm{~m} / \mathrm{s}$ \\
Boundary layer thickness $\delta$ & $38.1 \mathrm{~mm}$ \\
Wall friction velocity $u_{\tau}$ & $0.022 \mathrm{~m} / \mathrm{s}$ \\
Inner length scale/(Wall unit, WU) $\frac{v}{u_{\tau}}$ & $0.0467 \mathrm{~mm}$ \\
$R e_{\theta}=U_{\mathrm{e}} \theta / v$ & 2460 \\
$\operatorname{Re}_{\tau}=u_{\tau} \delta / v$ & 800 \\
Increments of vectors in & $0.6872 \mathrm{~mm}(14.7 \mathrm{WU})$ \\
$\quad x, y$, and $z$ direction & \\
\hline
\end{tabular}

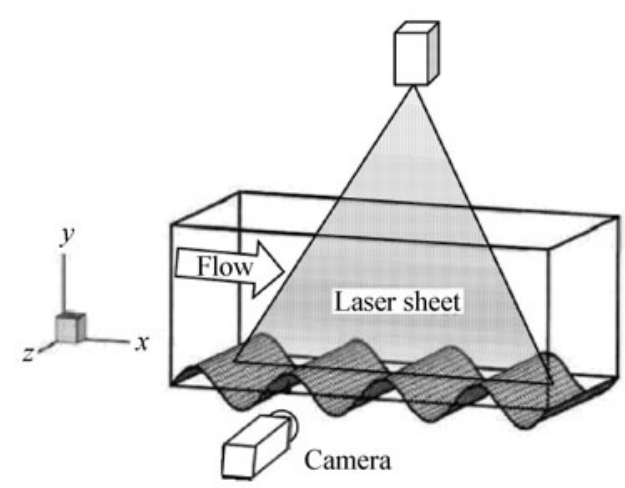

Fig. 1 The schematic of experimental measurement of TBL over a wavy wall

\section{Space-time correlations for turbulent boundary layer over a flat plate}

\subsection{Space-time correlation}

In the present paper, we are concerned only about the spacetime correlation of the streamwise velocity fluctuation based on the definition of
$C(r, \tau ; y)=\frac{\overline{u^{\prime}(x, y, z, t) u^{\prime}(x+r, y, z, t+\tau)}}{u_{\mathrm{rms}}^{\prime}(x) u_{\mathrm{rms}}^{\prime}(x+r)}$,

where $u^{\prime}$ is the streamwise fluctuation velocity and $u_{\mathrm{rms}}^{\prime}$ is the r.m.s of the streamwise fluctuation velocity. Note that instead of space-time correlation function $R(r, \tau)$, we use correlation coefficient $C(r, \tau)$ which is the normalized $R(r, \tau)$. In Eq. (4), the ensemble is implemented in streamwise, spanwise directions and time. Figure 2 is the $2 \mathrm{D}$ contours (right column) and 3D iso-surfaces (left column) of the space-time correlation at $y^{+}=56.8, y^{+}=130$, and $y^{+}=203$. The results display the behavior of the fluctuation velocity all in the logarithmic layer due to the limited wall-normal range of measurement. In the 3D iso-surfaces of the space-time correlations, the $x$-axis is the time delay $\tau^{+}$normalized by the inner time scale, the $y$-axis is the space separation $r^{+}$normalized by the inner length scale, and so do the axes in the $2 \mathrm{D}$ contours case, while the $z$-axis is the value of the spacetime correlation, which is from 0.5 to 0.9 with the increment of 0.05 . The range and increment of the three axes are the same in all plots at the three wall-normal locations. From the plots of the space-time correlation functions, the correlations behave in such a way that as the space separation and time delay increase, the magnitudes of correlations decrease because of the de-correlation of the small-scale motions, in contrast, when the space separation and time delay are small, the flow maintains a high level of correlation. Another character is revealed that far away from the wall, the coherent motions at the fixed correlation level increase both at the spatial and temporal scale, which will be seen more clearly from the 2D contours of space-time correlation shown in right column. This implies that the coherent structures grow within wider space and last for longer time. Adrian et al. [14] demonstrated that hairpins, hairpin packets and the uniform momentum zones induced by both the formers were actually prevalent in the logarithmic layer of turbulent boundary layers. As hairpins and hairpin packets moved downstream, the heads of the vortices continuously lifted up, the necks and legs were stretched, the packets contained more and more hairpins, and the zones of uniform momentum became larger [15], so the coherent structures increased their scales. The scenarios in this layer supported the above description about the space-time correlation functions. Moreover, the 3D space correlation results of Schröder et al. [13] showed the adjacent spanwise distributions of negative correlations, which were the manifestation of the lowspeed regions induced by hairpins and hairpin packets, and the inclinations of the coherent structures towards downstream. Both are consistent with the hairpin model. Kim et al. [16], Ganapathisubramani [17, 18] and Zhou et al. [19] have also described the scale growth of the correlation with the increasing $y$-locations.

From Fig. 2, it is obvious that the correlation functions have an elliptic shape. The distributions of the correlation functions follow the elliptic model of space-time correlation. The fact that the distributions of the space-time corre- 
lations are different from Taylor frozen hypothesis confirms the doubt that Taylor frozen hypothesis is not an appropriate description for the turbulent shear flow. The elliptic model of the space-time correlation correctly and successfully captures the de-correlation process which is not present in Taylor frozen hypothesis model.

\subsection{Characteristic parameters}

The distributions of the space-time correlation have two major characters [20,21]. One is that all the elliptic-like isocorrelation lines share a uniform preferred orientation. The uniform preferred orientation implies the association between the elliptic model and the Taylor frozen hypothesis model. The slope of the preferred orientation is mainly determined by the convection velocity $U_{\mathrm{c}}$ in both two models. Several schemes had been proposed to define the convection velocity. The convection velocity is usually derived from the frequency-wavenumber spectrum or its corresponding inverse Fourier transformation, i.e., the space-time correlation. One way is adopted by Wills [22], in which he searched for the maximum of the frequency spectrum along a constant wavenumber, or say, the maximum for the time correlation at a given space separation. Alternative way is adopted by Hussain et al. [23] and Goldschmidt et al. [24], in which they searched for the maximum of the wavenumber spectrum along a constant frequency, or say, the maximum
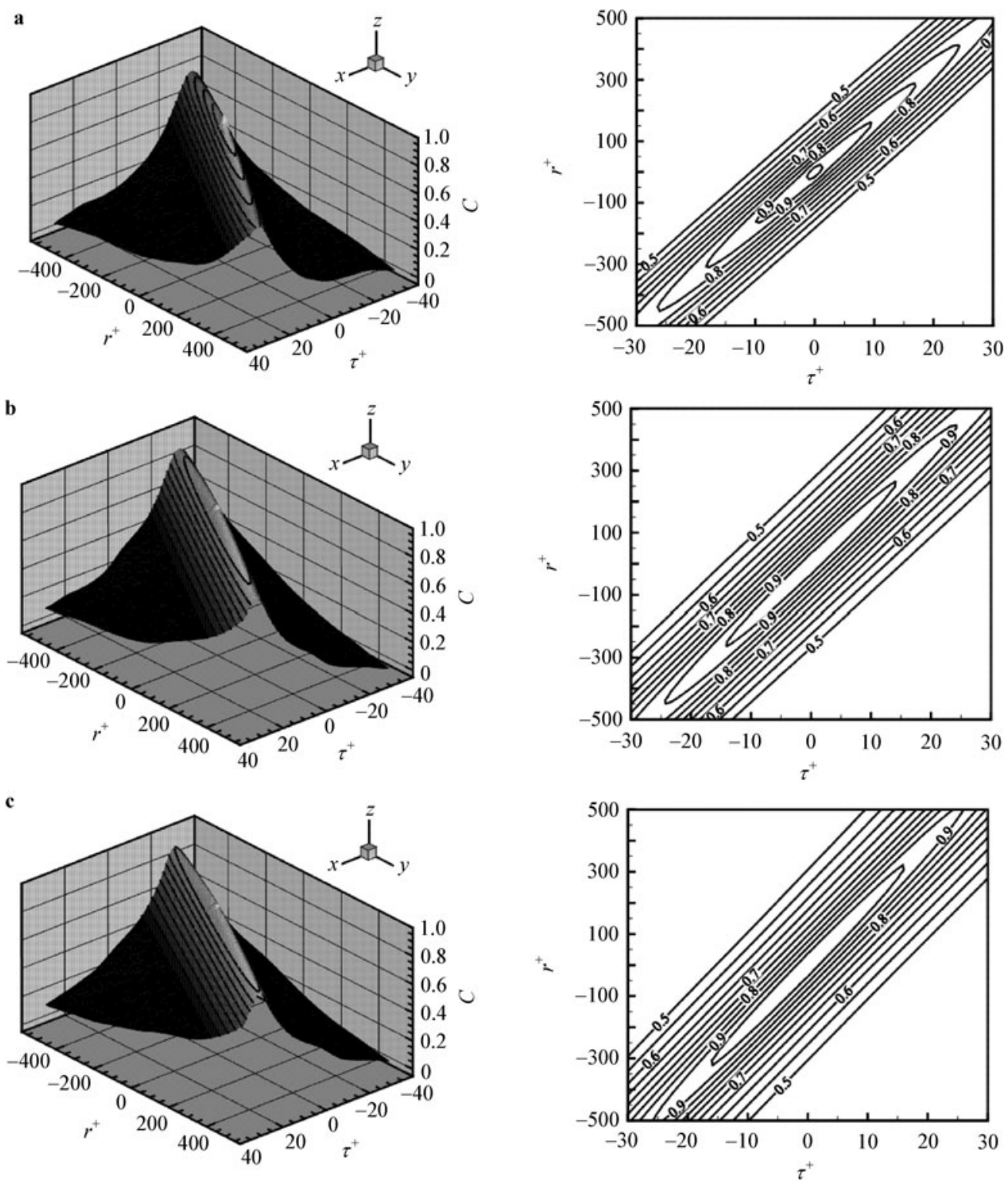

Fig. 2 The 3D surfaces (left column) and 2D contours (right column) of the space-time correlation with respect to time delay and space separation at $\mathbf{a} y^{+}=56.8 ; \mathbf{b} y^{+}=130 ; \mathbf{c} y^{+}=203$ 
for the space correlation at a given time delay. From Eq. (3), we can determine the convection velocity $U_{\mathrm{c}}$ based on

$\left.\frac{\partial r_{\mathrm{E}}}{\partial r}\right|_{\tau}=0, \quad r^{*}=U_{\mathrm{c}} \tau$,

where $r^{*}$ maximizes the correlation coefficient $C(r, \tau)$ for a given $\tau$. After the determination of each $r^{*}$ corresponding to each $\tau$, the convection velocity $U_{\mathrm{c}}$ is estimated either by averaging all the $r^{*} / \tau$, referred to as "the local mean $U_{\mathrm{c}}$ " or by linear fitting the slope of $r^{*}(\tau)$, referred to as "the linear fit $U_{\mathrm{c}}$ ". Figure 3 shows the estimated convection velocity $U_{\mathrm{c}}$ at $y^{+}=56.8, y^{+}=103$, and $y^{+}=203$. Figure $3 \mathrm{a}$ is the local mean $U_{\mathrm{c}}$ at $y^{+}=56.8$ and Figs. $3 \mathrm{~b}-3 \mathrm{~d}$ is the linear fit $U_{\mathrm{c}}$ at three $y^{+}$locations, respectively. Solid line in Fig. $3 \mathrm{a}$ is the local average value of the scattered $U_{\mathrm{c}}$ (hallow square symbols) and solid line in Figs. 3b-3d is the linear fit slope of $r^{*}(\tau)$. The linear fit $U_{\mathrm{c}}$ at $y^{+}=56.8$ is $0.3677 \mathrm{~m} / \mathrm{s}$, which is close to the local mean $U_{\mathrm{c}}$ of $0.3682 \mathrm{~m} / \mathrm{s}$; it is $0.4004 \mathrm{~m} / \mathrm{s}$ at $y^{+}=103$ and $0.4291 \mathrm{~m} / \mathrm{s}$ at $y^{+}=203$. Figure 4 is the convection velocity $U_{\mathrm{c}}$ calculated by the above methods from $y^{+}=56.8$ to $y^{+}=276.2$. In Fig. 4 the solid square is the average $U_{\mathrm{c}}$ with different $\tau$ and $r^{*}$, the hallow diamond is the linear fit $U_{\mathrm{c}}$ and the solid line is the local mean streamwise velocity $U$. The convection velocities $U_{\mathrm{c}}$ resulting from
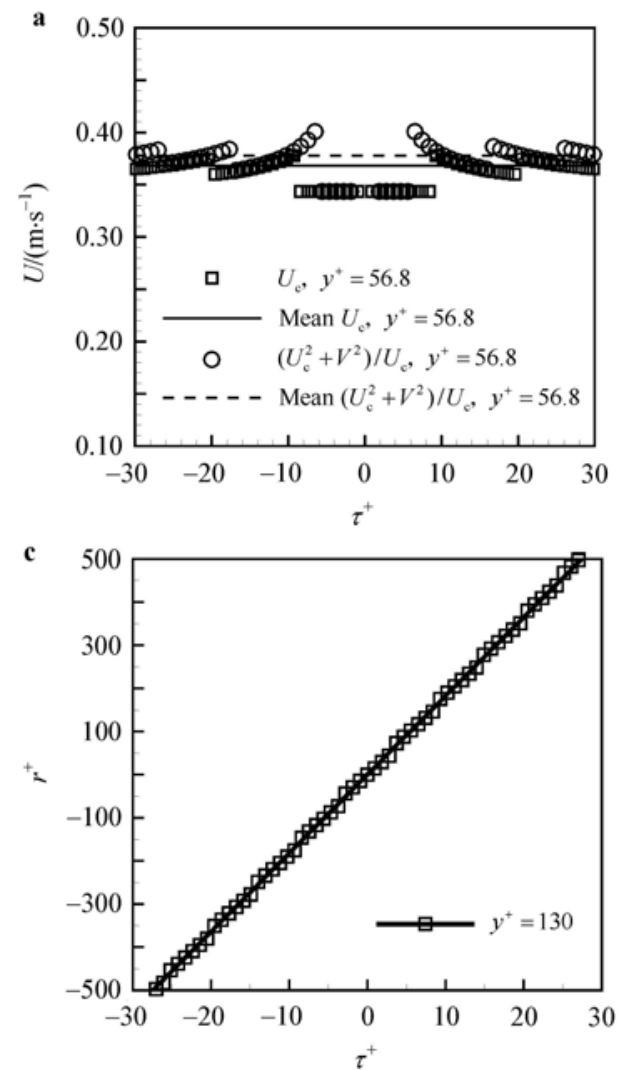

the two methods almost collapse together, so hereafter the convection velocity $U_{\mathrm{c}}$ will use the results by the linear fit method only. Away from the wall the convection velocity $U_{\text {c }}$ approaches the local mean velocity $U$. It is implied that the convection velocity $U_{\mathrm{c}}$ is associated and scaled with the mean flow. Kim et al. [16], Krogstad et al. [25], Del Alamo et al. [26] and Zhou et al. [10] all revealed this scaling property of the convection velocity $U_{\mathrm{c}}$ in the outer layer of the flow, while in the inner layer the two velocities were found not consistent with each other. In the elliptic model of the space-time correlation, Zhao et al. [7] theoretically proved that in a homogeneous shear flow the convection velocity $U_{\mathrm{c}}$ could be expressed as $U_{\mathrm{c}}=U$ where $U$ is the mean longitudinal velocity. The large-scale eddies carry the small-scale eddies at the convection velocity $U_{\mathrm{c}}$ in the flow and considerably contributed to the convection velocity $U_{\mathrm{c}}$. As the large-scale motions lift in height, the discrepancy between the convection velocity $U_{\mathrm{c}}$ and the mean velocity $U$ vanishes. It is implied that the scales of the coherent motions gradually transit to the outer layer scale $\delta$ and $U_{\mathrm{e}}$. The convection velocity $U_{\mathrm{c}}$ changes from $0.68 U_{\mathrm{e}}$ at $y^{+}=56.8$ to $0.85 U_{\mathrm{e}}$ at $y^{+}=276.2$, consistent with the range of the convection velocity in Ref. [14]. In their work, in the flow field multihairpin packets frequently coexisted, the old larger hairpin
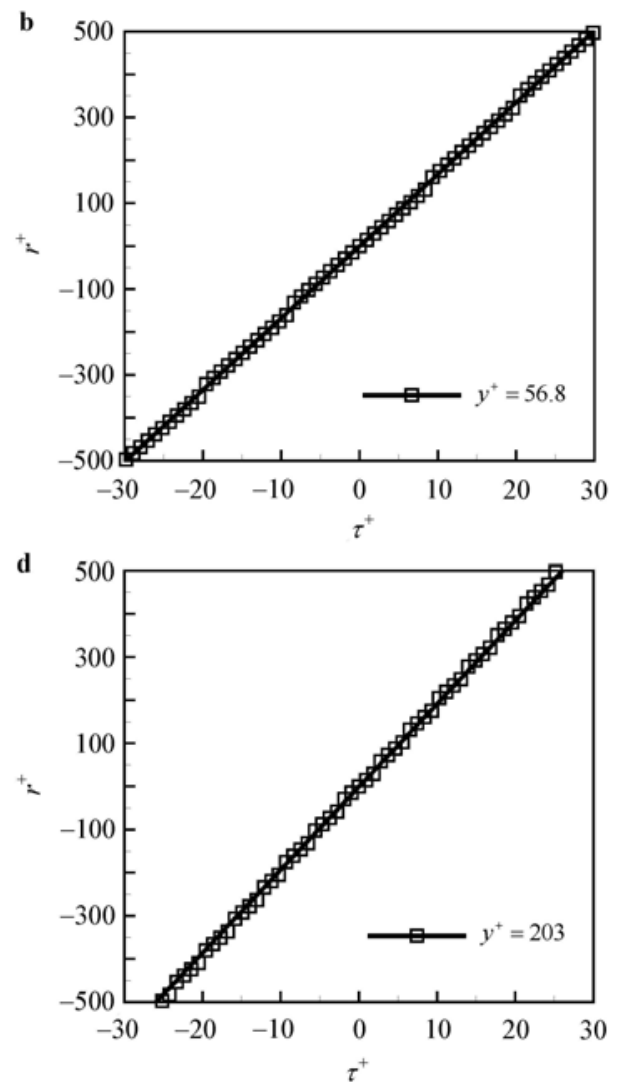

Fig. 3 The preferred orientation of the correlation contours as functions of time delays. a The convection velocity and sweep velocity estimated by mean method at $y^{+}=56.8$, where the solid and dashed lines denote the mean convection velocity and sweep velocity, respectively; b The convection velocity estimated by linear fit method at $y^{+}=56.8$; $\mathbf{c}$ The convection velocity estimated by linear fit method at $y^{+}=130 ; \mathbf{d}$ The convection velocity estimated by linear fit method at $y^{+}=203$ 


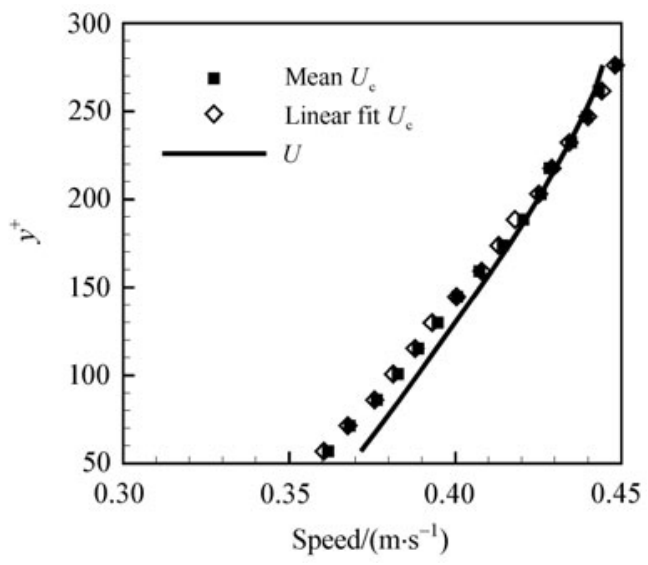

Fig. 4 The convection velocity calculated by two methods along the wall-normal direction. Solid square denotes the convection velocity resulting from the local average method. Hallow diamond denotes the convection velocity resulting from the linear fit method. The solid line denote the mean streamwise velocity in the flow

packets occupied higher locations meanwhile the young smaller hairpin packets moved at lower locations. The lower hairpin packets moved slower usually at $0.6 U_{\mathrm{e}}-0.7 U_{\mathrm{e}}$ while the higher hairpin packets moved faster at $0.8 U_{\mathrm{e}}-0.9 U_{\mathrm{e}}$. As the hairpin packets convected downstream, they were stretched and lifted up, resulting in larger convection velocity.

Another important characteristic velocity in Eq. (2) is the sweep velocity $V$, which can be determined based on

$\left.\frac{\partial r_{\mathrm{E}}}{\partial \tau}\right|_{r}=0, \quad \tau^{*}=\frac{U_{\mathrm{c}}}{U_{\mathrm{c}}^{2}+V^{2}} r$

where $\tau^{*}$ maximizes the correlation coefficient $C(r, \tau)$ for a given $r$. The sweep velocity $V$ is linearly fitted by the similar procedure mentioned above. The resulting sweep velocity $V$ in the $y$-direction is shown in Fig. 5. In the homogeneous shear flow case, Zhao et al. [7] claimed that the sweep velocity was intimately associated with the properties of the small scale eddies, such as Taylor micro-scale and random sweep velocity $[8,9]$ which represented the velocity fluctuations. They found that in the near wall region the shear rate and Taylor micro-scale had a significant contribution to the sweep velocity $V$, but in the logarithmic layer the velocity fluctuations dominated the sweep velocity $V$. Although the present turbulent boundary layer flow is more complex than homogeneous shear flow, the distribution of the sweep velocity $V$ follows the tendency of the streamwise fluctuation velocity mentioned in Ref. [13]. In the plots, the sweep velocity $V$ decreases with increasing vertical location in a fashion like the fluctuation velocity in the logarithmic layer. This supports the elliptic model of the space-time correlation.

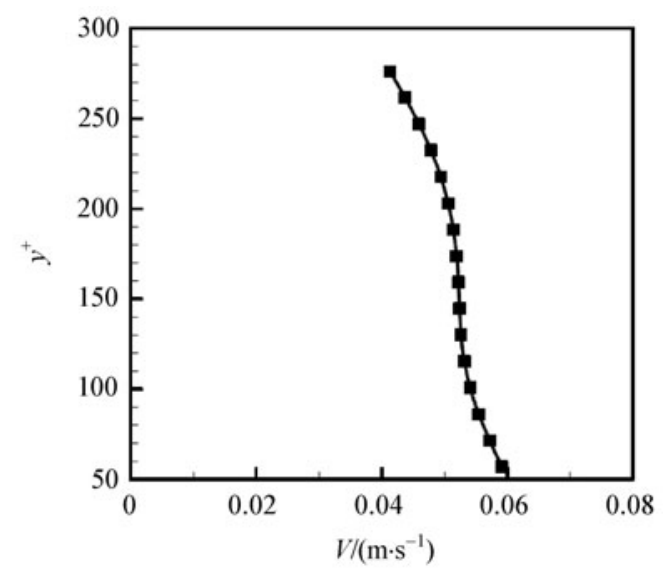

Fig. 5 The sweep velocity $V$ as a function of $y^{+}$in turbulent boundary layer

Another prominent character of the space-time correlation is the constant aspect ratio of the iso-correlation contours, which is defined as the length ratio of the major axis to the minor axis in the contour. The length of the major axis is the largest distance between two points on the iso-correlation contours, and the length of the minor axis is the least distance between them in contrast. The slopes in Fig. 6 represents the linear fit aspect ratios of the iso-correlation contours at $y^{+}=56.8, y^{+}=103$, and $y^{+}=203$, respectively. The constant aspect ratio can be derived from the Kolmogorov similar hypothesis [6]. While the preferred orientations of the contours is influenced by the convection velocity $U_{\mathrm{c}}$, the aspect ratio is mainly determined by the sweep velocity $V$. From Fig. 2, it is seen that at a given wall normal location, the contours of the correlation have a constant aspect ratio, and the contours become more elongated in the major axis as the location goes upwards. The aspect ratio in Fig. 6 indicates that the aspect ratio of the elliptic contours of correlation increases with increasing height. This confirms the relationship (6) between the aspect ratio and the sweep velocity $V$.

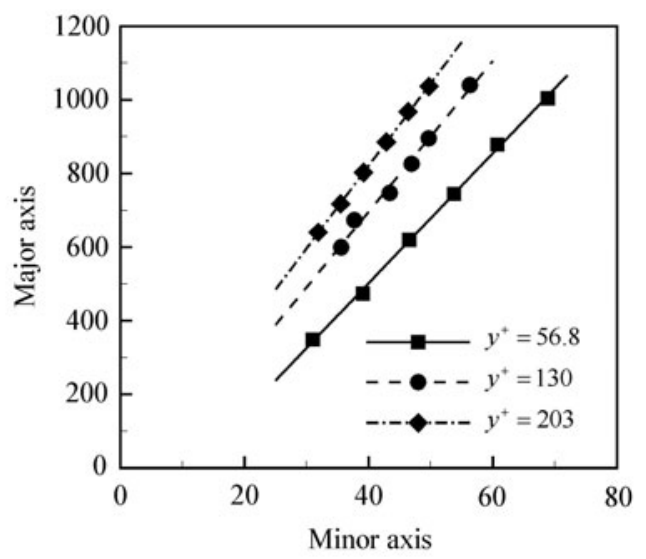

Fig. 6 The aspect ratios of the iso-correlation contours at $y^{+}=56.8$, 130, 203 


\subsection{Verification}

In this section we will verify the validity of the elliptic model of the space-time correlation by comparing it with the Taylor model. In the verification, we first plot the space correlations for different time delay $\tau^{+}=0,4.66,9.32,13.98$, 18.64 at $y^{+}=56.8,130,203$, respectively. Then we plot the corresponding correlation curves normalized by $r_{\mathrm{E}}$ - the space separation in the elliptic model and $r_{\mathrm{T}}$ - the space separation in the Taylor model, respectively. $r_{\mathrm{E}}$ is calculated by $r_{\mathrm{E}}=\sqrt{\left(r-U_{\mathrm{c}} \tau\right)^{2}+(V \tau)^{2}}$ and $r_{\mathrm{T}}$ is calculated by $r_{\mathrm{T}}=r-U \tau$. Owing to the symmetry of the correlation, we only plot below the part of positive space separation.

The leftmost column in Fig. 7 is the original space correlations, which display the evolution of the space-time correlation along time and space separation. Each correlation curve initially increases to the maximum and then drops off because the large-scale motions induce distortions of the small-scale eddies and accelerate the de-correlation process.
The magnitude of the peak decreases with time due to the evolution of the coherent structures, such as the spanwise meandering of the low speed streaks, the lift-up of hairpin vortices or the interactions between structures [11,27]. The middle column in Fig. 7 is the elliptic model normalized correlation curves which satisfactorily collapse into a single curve. In contrast, the rightmost column in Fig. 7 is the Taylor model normalized correlation curves. The differences among the correlation curves occur in the small space separation. It is considered that small scale eddies are responsible for the correlation in small separation. So it means that Taylor hypothesis can not accurately reproduce the small-scale motions [28]. It is not surprised because the small scale eddies have intense distortions forced by the large scale eddies and surrounding environment. Such realistic phenomena limit the application of Taylor hypothesis. Alternatively, the normalized correlation curves indicate that the elliptic model of the space-time correlation is more appropriate for the turbulent shear flow.

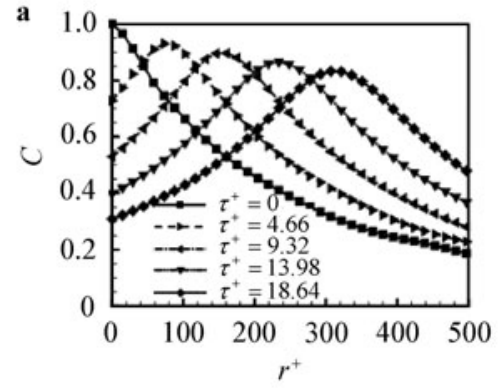

b
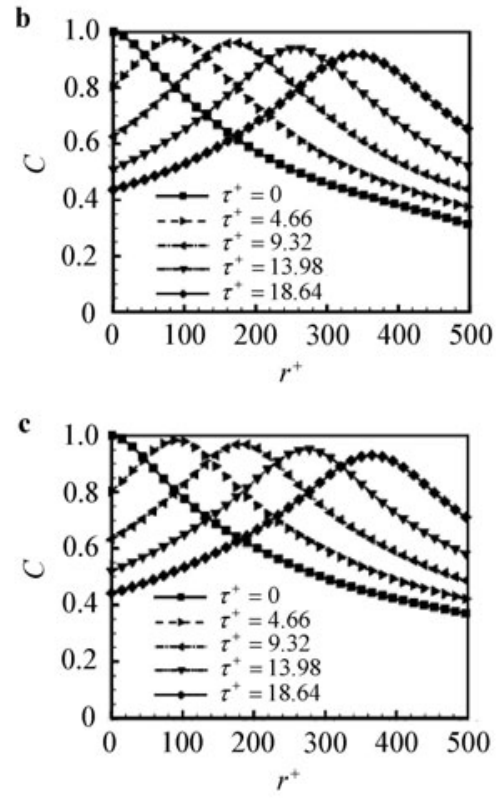
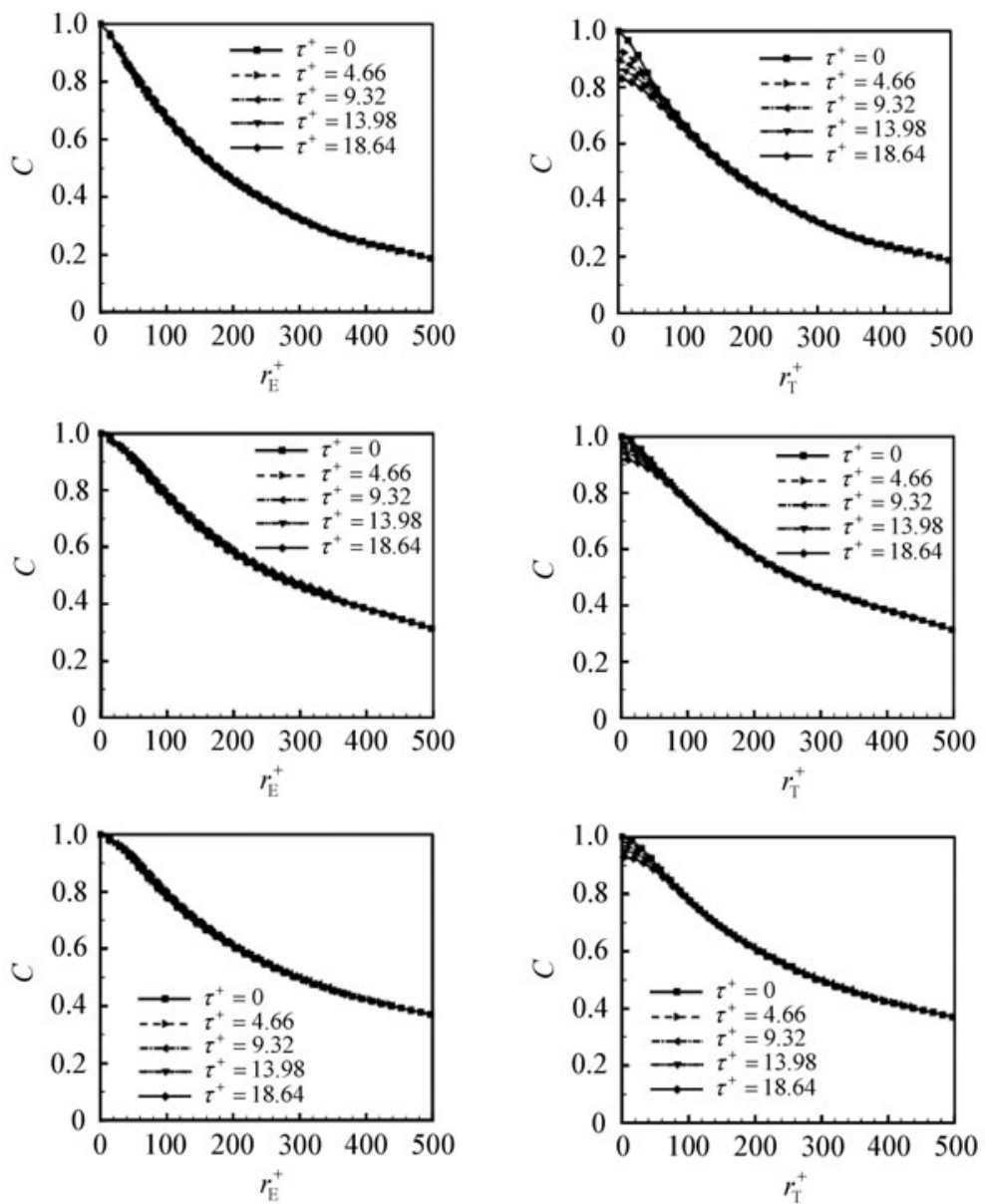

Fig. 7 The space-time correlation curves for 5 different time delay $\tau^{+}=0,4.66,9.32,13.98,18.64$ are plotted against space separation $r^{+}$(leftmost column), $r_{\mathrm{E}}^{+}$defined from the elliptic model and $r_{\mathrm{T}}^{+}$defined from Taylor hypothesis, respectively, at a $y^{+}=56.8$; $\mathbf{b} y^{+}=130$; c $y^{+}=203$ 


\section{Space-time correlations for turbulent flow over a wavy wall}

The turbulent flow over a wavy wall has a distinctly different nature from that over a flat wall. For the canonical turbulent boundary layer flow, coherent structures such as quasistreamwise vortex [29-31] are considered as the key to the production and sustaining of turbulence $[32,33]$. For flows over a wavy wall, shear layers $[34,35]$ are the sources of energy and Reynolds stress, as observed by Buckles et al. [34]. Turbulence in these regions is controlled by diffusion of turbulent kinetic energy from shear layers [36]. The interaction of turbulence formed by shear layer with large velocity gradients results in a large turbulence production.

Unlike the canonical TBL over a flat wall, the definition of TBL thickness over a wavy wall is subtle. We consider the midpoint of the waviness in the wall-normal direction as the origin of the boundary layer, like reference [35]. Then velocity profiles were wave-averaged at 5 locations, i.e., 1 crest, 2 through and 2 midway. The nominal boundary layer thickness is $32.5 \mathrm{~mm}$. In the present experiment, the resulting wave-averaged mean velocity profile and the turbulent intensity profiles are shown in Fig. 8. In spite of the complexity introduced by the wavy wall, the velocity distribution still follows the classical logarithmic law. The logarithmic law in Fig. 8a is $u^{+}=\ln y^{+} / \kappa+B$. The friction velocity $u_{\tau}$ is estimated to be $0.019 \mathrm{~m} / \mathrm{s}$ using the Newton iterative method [37]. Here the Kármán constant $\kappa$ is 0.4 , but the constant $B$ is -6 , much less than that in a normal turbulent boundary layer. The waviness of the boundary can be considered as a kind of large-scale roughness in the flow. Under the influence of the large-scale roughness, the velocity distribution decline somewhat towards the wall. Figure $8 \mathrm{~b}$ represents the r.m.s of the streamwise and wall normal velocity fluctuations, respectively. The distinctive character from the canonical distributions of the velocity fluctuations in turbulent boundary layer can be seen from Fig. 8 b. The 2 peaks in the r.m.s profile of streamwise velocity fluctuations stem from the aforementioned shear layer.

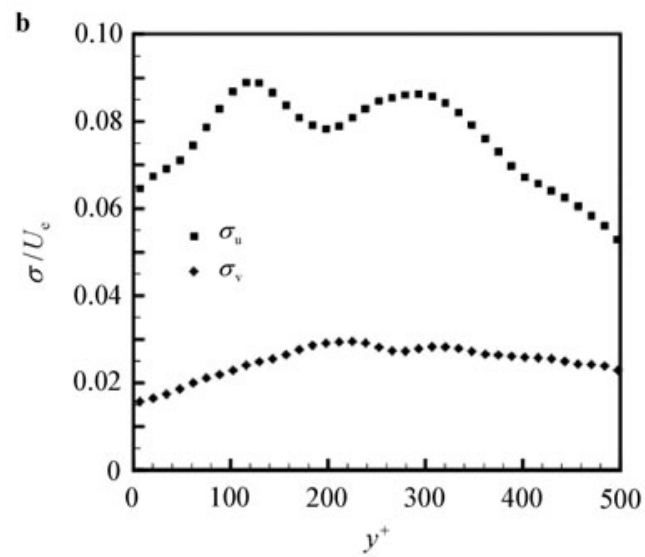

Fig. 8 a Wave-averaged streamwise velocity profile and $\mathbf{b}$ the r.m.s profiles of the streamwise and wall-normal velocity fluctuations in flow over a wavy wall

Shear layers were formed by the flow behind the wave crest and could extend over the wavelength of the wavy wall. Shear layers are defined as the locus of the streamwise turbulence intensity as used by Buckles et al. [34]. Figure 9 displays 2 shear layers develop over 2 wavelengths in the flow where the lower one locates at $y^{+} \approx 120$ and the upper one locates at $y^{+} \approx 300$. The evolutions of shear layers in the present work have a fashion similar to that reported in Buckles et al. [34], in which once shear layers were formed, they lifted slowly in a linear way along the flow direction.

The space-time correlations of the streamwise velocity fluctuation are calculated in the same way as Eq. (4) without spanwise direction. The 3D surfaces and $2 \mathrm{D}$ contours of the correlations are shown in Fig. 10, which displays space-time correlation at $y^{+}=6.8,115.6,278.8$, respectively. It is seen that while in the vicinity of the wave crest the shape of the correlation can be recognized as an ellipse, the contour of the

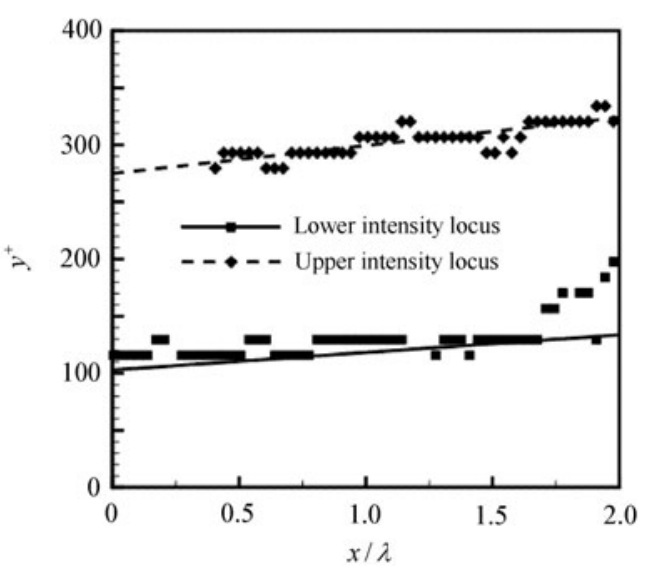

Fig. 9 Two shear layers defined as the locus of the local turbulent intensity maximum in flow over a wavy wall 
of the space-time correlation at $y^{+}=115.6$ do not show an elliptic shape at all but seems more like a triangle shape. Moreover the correlations at $y^{+}=115.6$ have higher correlation level and wider temporal-spatial scale. It is noticed that $y^{+}=115.6$ is within the region where the shear layer locates. The high magnitude of the correlation can be explained by the fact that the shear layer can last over long distance and long time. The shear layer even leave an imprint on the statistics of velocity fluctuation and maintain a great degree of coherence, resulting in a weakened decorrelation process which should be included in the elliptic model. Moving further away from the wall, the elliptic shape of the space-time correlation recurs in the outer region of the flow despite the shear layer. Comparing the influences from
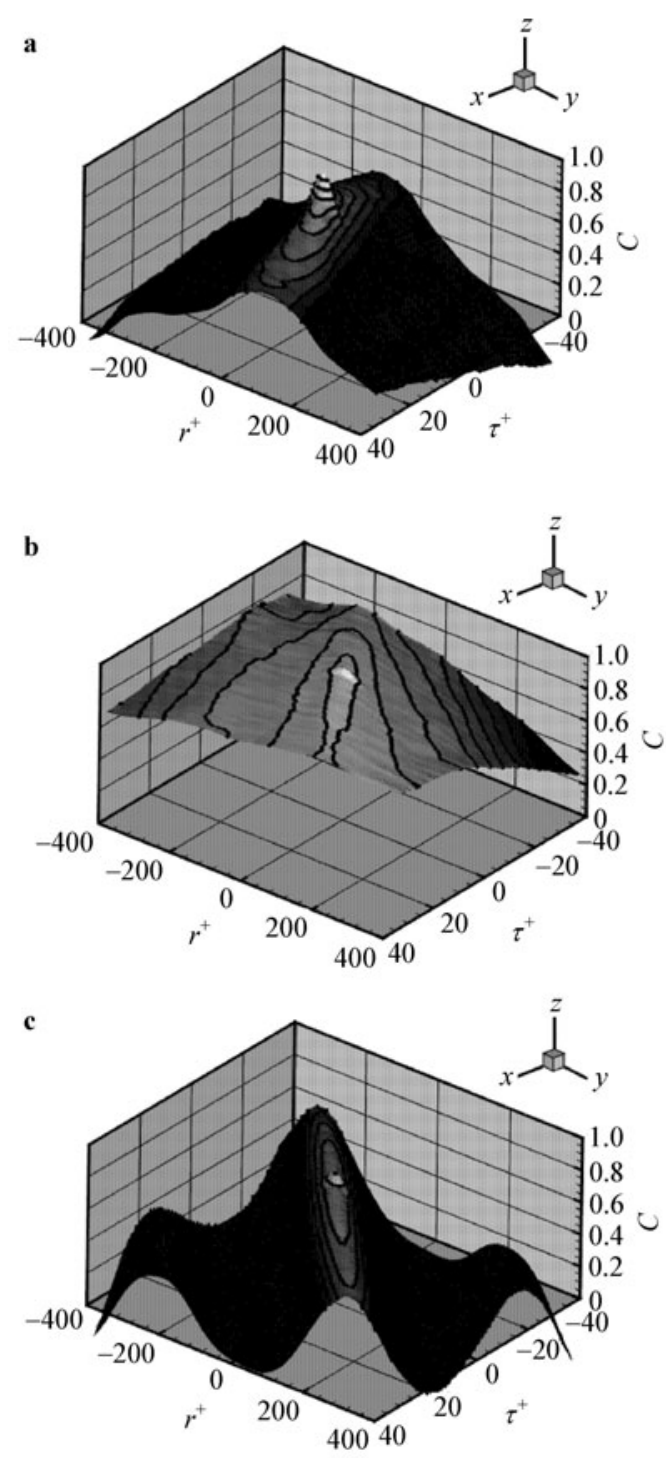

the lower and higher shear layers, it is considered that beside the shear layer the adverse pressure gradient [38-42], which is stronger near the wavy wall, may play an additional role in the invalidity of the elliptic model of the space-time correlation.

From the space-time correlation, the convection velocity $U_{\mathrm{c}}$ and the sweep velocity $V$ are respectively estimated using Eqs. (5) and (6). The resulting distributions are present in Fig. 11. Like the prediction of the elliptic model of the space-time correlation, in most regions of the flow the convection velocity $U_{\mathrm{c}}$ varied in response to the mean velocity $U$, while the sweep velocity $V$ is associated with the turbulent fluctuations. The differences of distributions between the flow over a wavy wall and that over a flat wall occurred
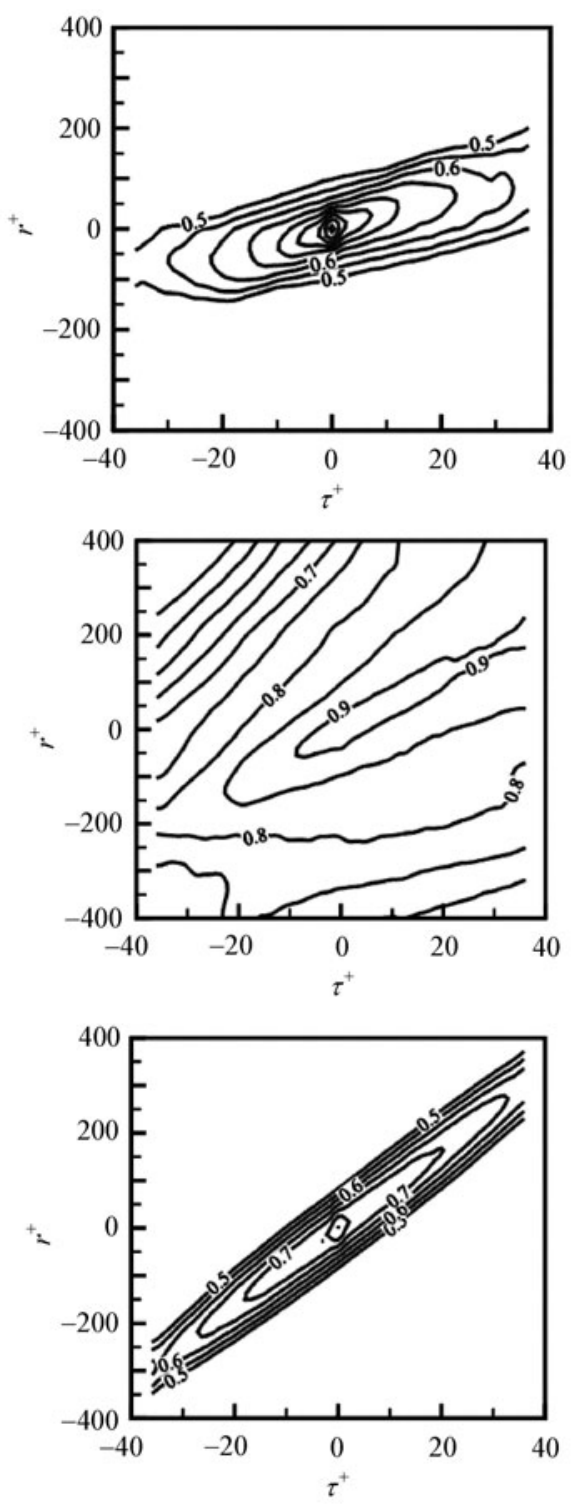

Fig. 10 The 3D surfaces (left column) and 2D contours (right column) of the space-time correlation with respect to time delay and space separation at $\mathbf{a} y^{+}=6.8 ; \mathbf{b} y^{+}=115.6 ; \mathbf{c} y^{+}=278.8$. In the $2 \mathrm{D}$ plots, the range of the correlation contours is from 0.5 to 0.9 with an increment of 0.05 
mainly within shear layers. According to the turbulent intensity distributions, the fluctuations were strongest within shear layers, which means that the small-scale motions overtake the large-scale motions due to the intensive shear effects. The convection velocity $U_{\mathrm{c}}$ dropped to local minimum at the locations of shear layers, in contrast the sweep velocity
$V$ reached its local maximum there. Both behaviors indicate that the small-scale fluctuations decrease the convection velocity and increase the sweep velocity.

After knowing the impact of shear layers, it is critical to verify the elliptic model and the Taylor model. Figure 12 illustrates the original correlation curves, the correlation
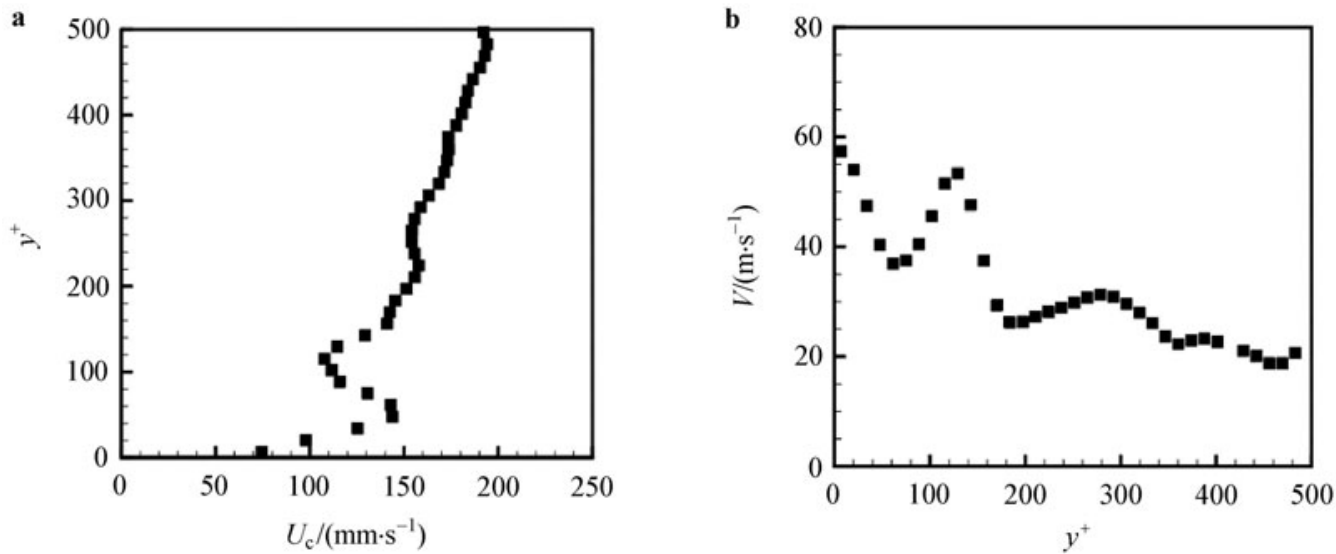

Fig. 11 a The convection velocity and $\mathbf{b}$ the sweep velocity along the wall-normal direction in flow over a wavy wall
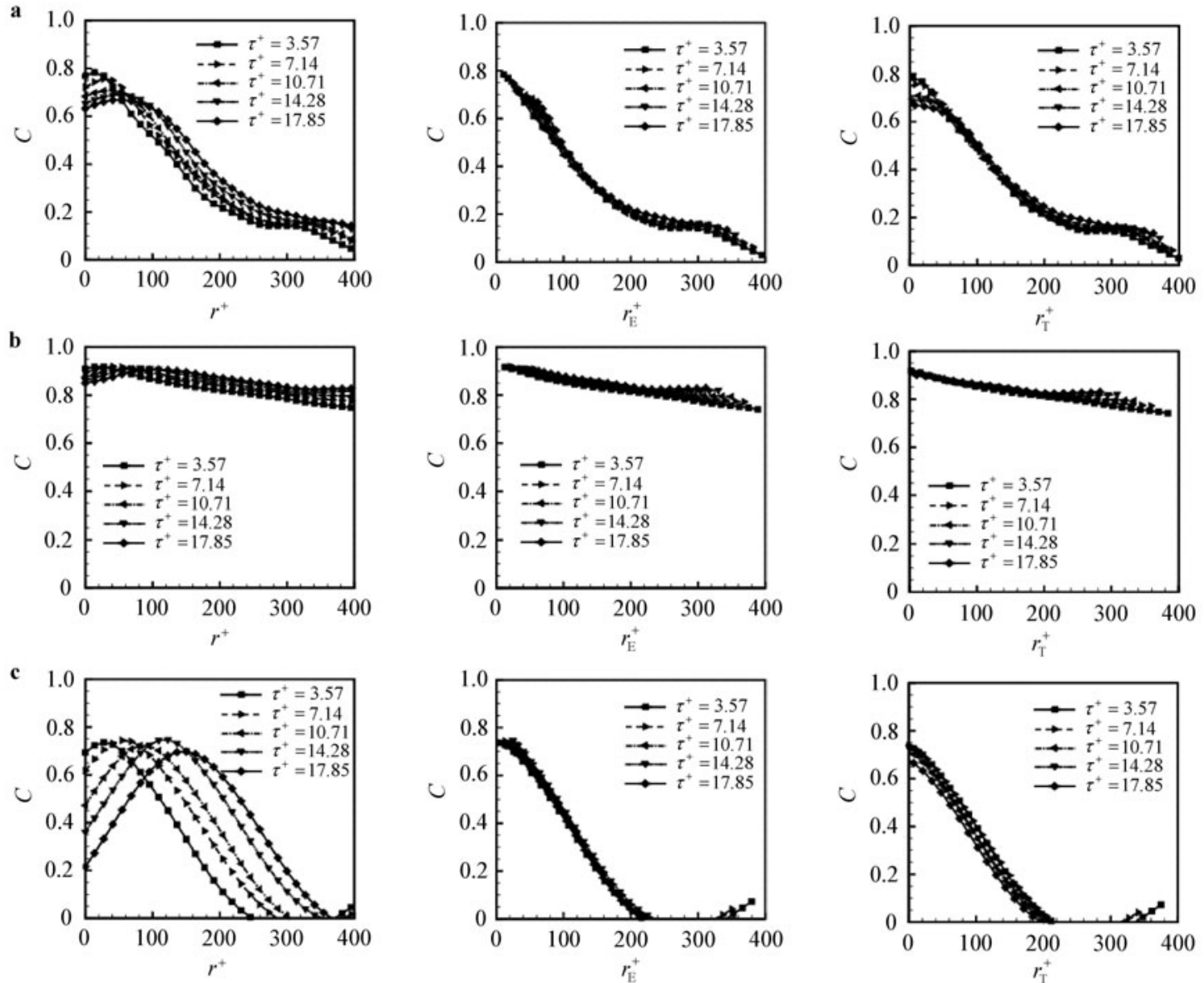

Fig. 12 The space-time correlation curves for 5 different time delay $\tau^{+}=3.57,7.14,10.71,14.28,17.85$ are plotted against space separation $r^{+}$(leftmost column), $r_{\mathrm{E}}^{+}$defined from the elliptic model and $r_{\mathrm{T}}^{+}$defined from Taylor hypothesis respectively at a $y^{+}=6.8$; b $y^{+}=115.6 ; \mathbf{c} y^{+}=278.8$ 
curves normalized with the elliptic model and the correlation curves normalized with the Taylor model at the same three heights. It is expected that the more the space-time correlation accords with the elliptic model at the height, the greater the curves fit the elliptic model. The curves normalized by the elliptic model coincide each other better than those by the Taylor model at $y^{+}=6.8$ and $y^{+}=278.8$, where the iso-correlation contours indeed had an elliptic shape. But at $y^{+}=115.6$, neither curves normalized by the elliptic model nor those by the Taylor model collapse together, since the elliptic model of the space-time correlation is neither valid here. In a word, the elliptic model of the space-time correlation may be invalid in some specific flow situations, but once the elliptic model is valid, it describes the space-time correlation better than the Taylor model.

\section{Conclusions}

The 3D Tomo-TRPIV and 2D planar-TRPIV were applied to an instantaneous velocity measurement in turbulent boundary layers over a flat wall and a wavy wall, respectively. The space-time correlation of the fluctuation velocity is calculated and investigated. The contours of the space-time correlations show an elliptic shape which supports the elliptic model. In the elliptic model, the convection velocity scales with the mean longitudinal velocity and the sweep velocity is intimately associated with the fluctuations of the velocity. The correlation curves normalized by the elliptic model coincide each other better than those by the Taylor model. It is thus concluded that the elliptic model of space-time correlation is more accurate than the Taylor hypothesis for turbulent shear flows. The flow over a wavy wall reveals some limitation of the new elliptic model. The shear layer with adverse pressure gradient undermines the validity of the elliptic model. In spite of the shear layer in the inner region, the elliptic model is found still valid in the outer region over a way wall. Besides the turbulent flows studied in the present work, the elliptic model should be verified in more flow situations in the future work. For an extensive investigation of the elliptic model, it is expected that the parameters in the elliptic model, $U_{\mathrm{c}}$ and $V$, will need a thorough understanding concerning their variations in turbulent flows, such as scaledependency, relevant turbulence quantities and so on.

Acknowledgements The authors sincerely thank all the members of the experimental team in TU Delft, LaVision GmbH and the German Aerospace Center (DLR) for providing the Tomographic PIV data.

\section{References}

1 Kolmogorov, A.N.: The local structure of turbulence in incompressible viscous fluid for very large Reynolds numbers. Dokl. Akad. Nauk SSSR. 30, 299-303 (1941)

2 Saric, W.S., Reed, H.L. Kerschen, E.J.: Boundary-layer receptivity to free-stream disturbance. Ann. Rev. Fluid Mech. 34, 291 (2002)
3 Lumley, J., Blossy, P.: Control of turbulence. Ann. Rev. Fluid Mech. 30, 311 (1998)

4 Taylor, G.I.: The spectrum of turbulence. Proc. R. Soc. Lond. 164, 476-490 (1938)

5 Lin, C.C.: On Taylor's hypothesis and the acceleration terms in Navier-Stokes equation. Q. Appl. Math. 10, 295 (1953)

$6 \mathrm{He}$, G.W., Zhang, J.B.: Elliptic model for space-time correlations in turbulent shear flows. Phys. Rev. E. 73, 055303 (2006)

7 Zhao, X., He, G.W.: Space-time correlations of fluctuating velocities in turbulent shear flows. Phys. Rev. E. 79, 046316 (2009)

8 Kraichnan, R.H.: Kolmogorov's hypotheses and Eulerian turbulence theory. Phys. Fluids 7, 1723 (1964)

9 Tennekes, H.: Eulerian and Lagrangian time micro-scales in isotropic turbulence. J. Fluid. Mech. 67, 561-567 (1975)

10 Zhou, Q., Li, C.M., Lu, Z.M., et al.: Experimental investigation of longitudinal space-time correlations of the velocity field in turbulent Rayleigh-Bénard convection. J. Fluid Mech. 683, 94-111 (2011)

11 Elsinga, G.E., Wieneke, F., van Oudheusden, B.W.: Tomographic particle image velocimetry. Exp. Fluids. 41, 933-947 (2006)

12 Wieneke, B.: Volume self-calibration for 3D particle image velocimetry. Exp. Fluids 45, 549-556 (2008)

13 Schröder, A., Geisler, R., Staack, K., et al.: Eulerian and Lagrangian views of a turbulent boundary layer flow using timeresolved tomographic PIV. Exp. Fluids 50, 1071-1091 (2010)

14 Adrian, R.J., Meinhart, C.D., Tomkins, C.D.: Vortex organization in the outer region of the turbulent boundary layer. J. Fluid Mech. 422, 1-54 (2000)

15 Christensen, K.T., Adrian, R.J.: Statistical evidence of hairpin vortex packets in wall turbulence. J. Fluid. Mech. 431, 433443 (2001)

16 Kim J., Hussain F.: Propagation velocity of perturbations in turbulent channel flow. Phys. Fluids 5, 695-705 (1992)

17 Ganapathisubramani, B., Clemens, N.T., Dolling, D.S.: Largescale motions in a supersonic boundary layer. J. Fluid Mech. 556, 271-282 (2006)

18 Ganapathisubramani, B.: Statistical properties of streamwise velocity in a supersonic turbulent boundary layer. Phys. Fluids 19, 098108 (2007)

19 Zhou, J., Adrian, R.J., Balachandar, S., et al.: Mechanism for generating coherent packets of hairpin vortices in channel flow. J Fluid Mech. 387, 353-396 (1999)

$20 \mathrm{He}$, X.Z., He, G.W., Tong, P.E.: Small-scale turbulent fluctuations beyond Taylor's frozen-flow hypothesis. Phys. Rev. E. 81, 065303 (2010)

21 Guo, L., Li D., Zhang, X., He, G.W.: LES prediction of spacetime correlations in turbulent shear flows. Acta Mechanica Sinica 28, 993-998 (2012)

22 Wills, J.A.B.: On convection velocities in turbulent shear flows. J. Fluid Mech. 20, 417-432 (1964)

23 Hussain, A.K.M.F., Clark, A.R.: Measurements of wavenumber-celerity spectrum in plane and axisymmetric jets. AIAA J. 19, 51-55 (1981)

24 Goldschmidt, V.W., Young, M.F., Ott, E.S.: Turbulent convective velocities (broadband and wavenumber dependent) in a plane jet. J. Fluid Mech. 105, 327-345 (1981) 
25 Krogstad, P., Kaspersen, J.H., Rimestad, S.: Convection velocities in a turbulent boundary layer. Phys. Fluids. 10, 949-957 (1997)

26 Del Alamo, J.C., Jiménez, J.: Estimation of turbulent convection velocities and corrections to Taylor's approximation. J. Fluid Mech. 640, 5-26 (2009)

27 Tomkins, C.D., Adrian, R.J.: Spanwise structure and scale growth in turbulent boundary layers. J. Fluid Mech. 490, 37-74 (2003)

28 Dennis, D.J.C., Nickels, T.B.: On the limitations of Taylor's hypothesis in constructing long structures in a turbulent boundary layer. J. Fluid Mech. 614, 197-206 (2008)

29 Rodinson, S.K.: Coherent motions in the turbulent boundary layer. Annu. Rev. Fluid Mec. 23, 601-639 (1991)

30 Hussain, F.: Coherent structures-Reality and myth. Phys. Fluids 26, 2816-2850 (1983)

31 Schoppa, W., Hussain, F.: Coherent structure generation in near-wall turbulence. J. Fluid Mech. 453, 57-108 (2002)

32 Panton, R.L.: Overview of the self-sustaining mechanisms of wall turbulence. Progress in Aerospace Sciences 37, 341-383 (2001)

33 Brooke, J.W., Hanratty, T.J.: Origin of turbulence producing eddies in a channel flow. Phys. Fluids A 5, 1011-1022 (1993)

34 Buckles, J., Hanratty, T.J., Adrian, R.J.: Turbulent flow over large-amplitude wavy surfaces. J. Fluid Mech. 140, 27-44
(1984)

35 Hudson, J.D., Dykhno, L., Hanratty, T.J.: Turbulence production in flow over a wavy wall. Exp. Fluids. 20, 257-265 (1996)

36 Kruse, N., Kuhn, S., von Rohr, P.R.: Wavy wall effects on turbulence production and large-scale modes. Journal of Turbulence 7, 1-24 (2006)

37 Fan, X., Jiang, N.: Skin friction measurement in turbulent boundary layer by mean velocity profile method. Mechanics in Engineering. 27, 28-30 (2005)

38 Zilker, D.P., Cook, G.W., Hanratty, T.J.: Influence of the amplitude of a solid wavy wall on a turbulent flow Part 1. Nonseparated flows. J. Fluid Mech. 82, 29-51 (1977)

39 Zilker, D.P., Hanratty, T.J.: Influence of the amplitude of a solid wavy wall on a turbulent flow Part 2. Separated flows. J. Fluid Mech. 90, 257-271 (1979)

40 Kuzan, J.D., Hanratty, T.J., Adrian, R.J.: Turbulent flows with incipient separation over solid waves. Exp. Fluids 7, 88-98 (1989)

41 Cherukat, P., Na, Y., Hanratty, T.J., et al.: Direct numerical simulation of a fully developed turbulent flow over a wavy wall. Theoretical and Computational Fluid Dynamics 11, 109-134 (1998)

42 De Angelis, V., Lombardi, P., Banerjee, S.: Direct numerical simulation of turbulent flow over a wavy wall. Phys. Fluids 9, 2429-2442 (1997) 